\title{
Electrical storm induced by hypokalemia associated with herbal medicines containing licorice
}

\author{
Hyun Kuk Kim and Sung Soo Kim* \\ Division of Cardiology of Chosun University Hospital, Gwangju 61453, Korea \\ ${ }^{*}$ Correspondence: S. S. Kim; Tel: +82-62-220-3240, Fax: +82-62-228-7174, E-mail: kholywater@gmail.com
}

\section{Check for updates}

Received 5 Apr 2019

Revised 15 May 2019

Accepted 17 May 2019

Keywords

Arrhythmia,

Hypokalemia,

Licorice

pISSN: 2289-0882

elSSN: $2383-5427$
A 60-year-old woman presented with polymorphic ventricular tachycardia secondary to hypokalemia, which necessitated dozens of DC cardioversions. She was not taking any other medication and denied any vomiting or diarrhea. Further investigation for hypokalemia suggested a hypermineralocorticoid state. Repeated inquiry prompted the patient to admit to taking herbal medicine containing licorice. She was treated with magnesium sulfate, potassium infusion, and intravenous lidocaine. A potassium-sparing diuretic was also prescribed. On the seventh day, the patient was discharged from the hospital with advice to discontinue taking herbal medicines containing licorice. She has been followed up at our outpatient clinic without further symptoms for 3 years. This case highlights the potential for cardiovascular complications associated with consumption of herbal medicines such as licorice. Clinicians should be aware that patients presenting to the emergency department with ventricular arrhythmia and uncertain hypokalemia should be questioned about licorice intake. Obtaining a detailed history from patients admitted to the hospital for electrical storm is essential.

\section{Introduction}

Licorice is one of the most widely prescribed herbs in traditional Korean medicine. Life-threatening ventricular arrhythmia may be caused by hypokalemia associated with licorice consumption, presumably through inhibition of the cortisolconverting enzyme $11 \beta$-hydroxysteroid dehydrogenase.[1,2] Recently, we experienced a rare case of electrical storm secondary to hypokalemia caused by the intake of herbal medicine containing licorice.

\section{Case Report}

A 60-year-old woman presented at our emergency department complaining of chest discomfort. She had no family history of sudden cardiac death, or genetic disease. She was not taking any other medication and denied any vomiting or diarrhea.

Copyright ( 2019 Translational and Clinical Pharmacology

(a) It is identical to the Creative Commons Attribution Non-Commercial License (http://creativecommons.org/licenses/by-nc/3.0/).

@ This paper meets the requirement of KS X ISO 9706, ISO 9706-1994 and ANSI/NISO Z.39.48-1992 (Permanence of Paper).

Reviewer

This article was reviewed by the peer experts who are not TCP editors
On admission, she was hypotensive and the monitor showed an irregular polymorphic ventricular rhythm. Her vital signs were as follows: body temperature, $36.7^{\circ} \mathrm{C}$; pulse, 183 beats/ $\mathrm{min}$; breathing, 25/min; and blood pressure, 70/40 $\mathrm{mmHg}$. Twelve-lead ECG showed polymorphic ventricular tachycardia (Fig. 1A). She was defibrillated and resuscitation was started immediately. However, she developed incessant repetitive ventricular arrhythmia of different types (Fig. 1B) and seizure-like movement, which necessitated dozens of DC cardioversion and defibrillation over 3 hours. Coronary angiography to rule out coronary artery disease showed almost normal (Fig. 2). Portable echocardiography showed no structural heart disease and regional wall motion abnormality.

The results of the initial blood testing were as follows: white blood cell count, 9,560/ $\mathrm{mm}^{3}$; Hemoglobin, $10.9 \mathrm{~g} / \mathrm{dL}$; platelets, 232,000/. $\mathrm{mm}^{3}$; sodium $(\mathrm{Na}), 138 \mathrm{mmol} / \mathrm{L}$; potassium $(\mathrm{K}), 2.4$ $\mathrm{mmol} / \mathrm{L}$; chlorine, $105 \mathrm{mmol} / \mathrm{L}$; calcium $(\mathrm{Ca}), 9.65 \mathrm{mg} / \mathrm{dL}$ and magnesium (Mg), $2.48 \mathrm{mg} / \mathrm{dL}$.

Diagnostic workup for other causes of hypokalemia was performed. Her spot urinary K concentration was $20.7 \mathrm{mmol} / \mathrm{L}$ (normal, $<15 \mathrm{mmol} / \mathrm{L}$ ), which suggested inappropriate urinary $\mathrm{K}$ excretion. Her transtubular K gradient became 4.8, which 


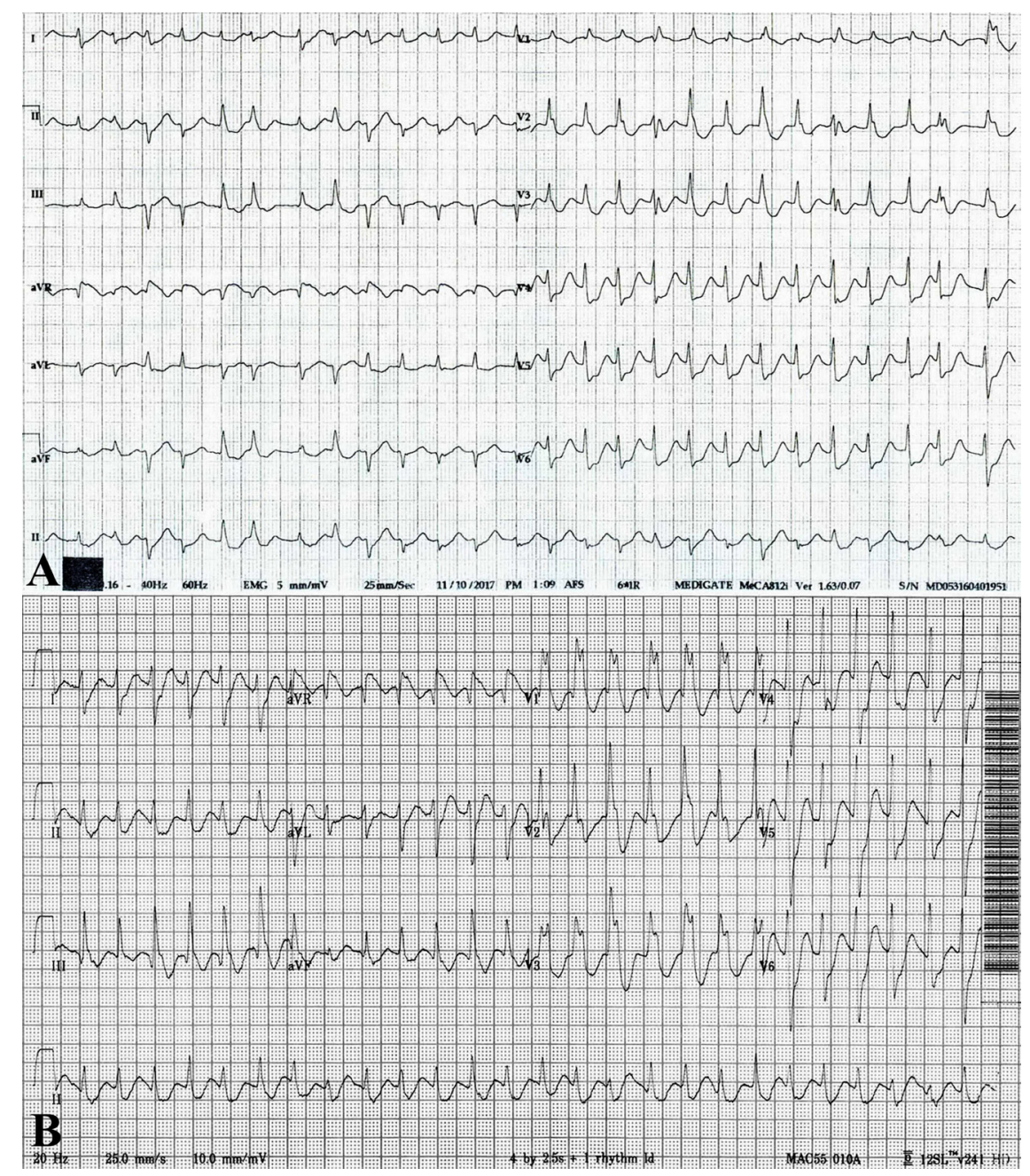

Figure 1. Recurrent sustained polymorphic ventricular tachycardia necessitating multiple DC cardioversion at emergency department. (A) 12 lead ECG showed polymorphic ventricular tachycardia. (B) 12 lead ECG showed monomorphic ventricular tachycardia with uniform RBBB morphology, RSR' complex with a taller left rabbit ear sign, $r S$ complex in V6 and AV dissociation.

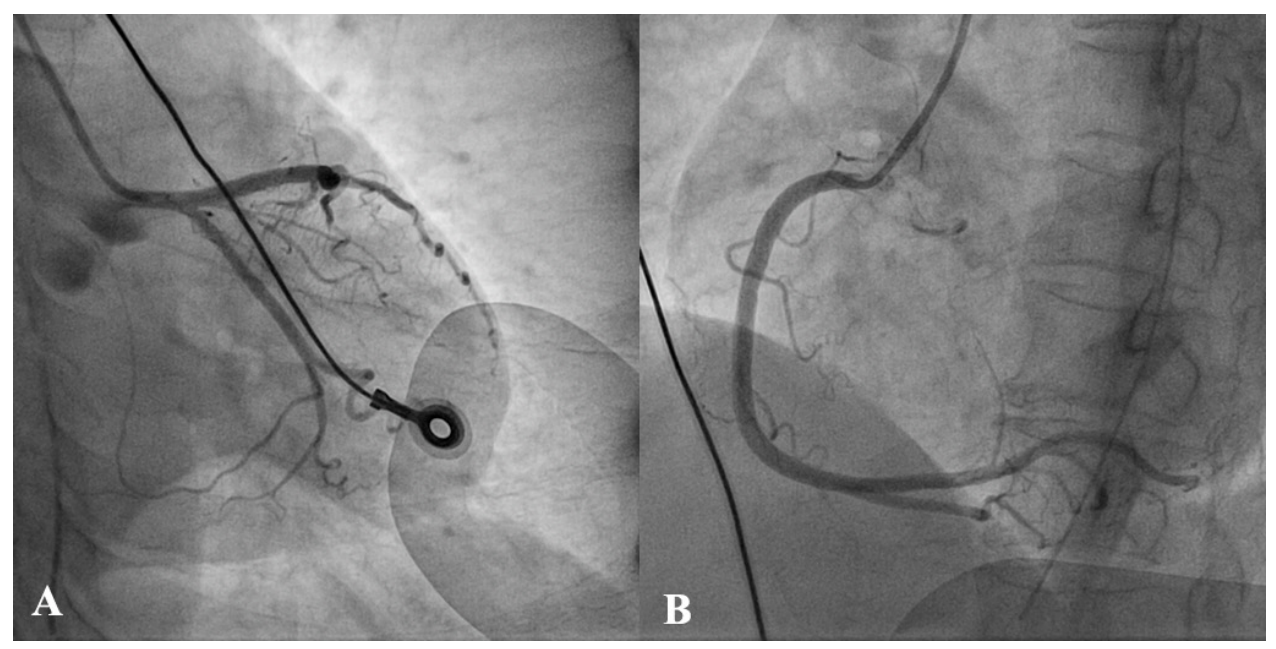

Figure 2. Coronary angiogram showed no significant stenosis in left anterior descending artery and left circumflex coronary artery (A) and right coronary artery (B). 
indicated increased distal tubular K secretion. The plasma aldosterone concentration was low $(0.6 \mathrm{ng} / \mathrm{dL}$; normal, $2.7-27.2 \mathrm{ng} /$ $\mathrm{dL})$ and plasma cortisol concentration was normal $(23.8 \mathrm{ug} / \mathrm{dL}$; normal, 5-27 ug/dL). These laboratory results suggested that the patient's hypokalemia was caused by a hypermineralocorticoid state.

The patient denied any use of medications such as diuretics or laxatives that are possible exogenous causes of hypokalemia. However, the patient revealed in a repeated medical history that, for the past several months, she had ingested herbal medicine containing licorice thrice daily for the treatment of herniated intervertebral disks. This history suggested to us that the patient had experienced licorice-induced hypokalemia with electrical storm.

She was treated with magnesium sulfate, $\mathrm{K}$ infusion, and intravenous lidocaine. A K-sparing diuretic was also prescribed. On the seventh day, the patient was discharged from the hospital with advice to discontinue ingesting herbal medicines containing licorice. She has been followed up at our outpatient clinic without further symptoms for 3 years. After discontinuing the use of licorice-containing medicines, the patient's $\mathrm{K}$ level was within the normal level $(4.3 \mathrm{mEq} / \mathrm{L})$ and 24 -hour Holter examination results were normal.

\section{Discussion}

Electrical storm is defined as a state of cardiac electrical instability that involves incessant recurrent ventricular arrhythmia. Most patients with electrical storm have severe underlying structural heart disease, and studies have revealed an inciting factor in only a minority of patients with electrical storm.[3] However, careful assessment is required as some of the known triggers are reversible, including drug toxicity, electrolyte imbalance, QT prolongation and myocardial ischemia. This patient developed severe hypokalemia secondary to licorice use, which led to incessant repetitive ventricular arrhythmia of different types. Ventricular rhythm with frequent variation of the alternating QRS axis, RBBB morphology (Fig. 1A). Another type was monomorphic VT with uniform RBBB morphology, RSR' complex with a taller left rabbit ear sign, rS complex in V6, and atrioventricular dissociation (Fig. 1B). Myopathy and rhabdomyolysis caused by hypokalemia due to licorice have been reported,[4-7] but ventricular arrhythmia represented by an electrical storm have never been reported in Korea. Only a few case reports have described this event in the western countries.[8-12]

Licorice has been used as a food additive (sweetener), in cosmetics, and in herbal remedies in traditional Korean medicine for a long time. The active component of licorice is glycyrrhetinic acid, which inhibits the enzyme $11 \beta$-hydroxysteroid dehydrogenase.[1] This enzyme promotes the conversion of cortisol to cortisone and is thereby responsible for the specificity of the mineralocorticoid receptor to aldosterone in the collecting tubules. Inhibition of the enzyme allows cortisol to act as the major endogenous mineralocorticoid producing a marked elevation in mineralocorticoid activity, causing hypertension, renal potassium loss, high bicarbonate and metabolic alkalosis. Therefore, it is common to be treated with hypertension drugs containing diuretic, which hypokalemia can become even worse. In the case of hypokalemia associated with licorice reported in Korea, most patients were taking the diuretics. $[6,13]$ However, in our case, she did not take diuretics and other medicines.

Hypokalemia can influence cardiac myocytes by hyperpolarizing the membrane electrical potential, which tends to open $\mathrm{Na}$ channels and not to open K influx channels. Hypokalemia also decreases the activity of rapid-activating delayed-rectifier channels, which play a role in repolarization. Hypokalemia itself is a well-known major risk factor for polymorphic ventricular arrhythmia.[11]

There is apparently a great individual variation in the susceptibility to licorice. Most individuals who consume $400 \mathrm{mg}$ glycyrrhizic acid daily experience adverse effects. [14] It can also occur by taking a small dose of glycyrrhizic acid $100 \mathrm{mg}$. The mechanism of this difference in susceptibility has not been clarified but is thought to be due to genetic mutation. Genetic mutation in the $11 \beta$-hydroxysteroid dehydrogenase type 2 (HSD11B2) gene may compromise its protective action against cortisol access to the mineralocorticoid receptor, and this might lead to greater sensitivity to licorice. Russo et al suggested that some people may be susceptible to low doses of licorice because of deficiency in $11 \beta$-hydroxysteroid dehydrogenase.[15] Harahap et al found that licorice ingestion and mutations in HSD11B2 inhibit $11 \beta$-hydroxysteroid dehydrogenase type 2 enzyme activity and cause the syndrome of apparent mineralocorticoid excess. [16] They speculated that licorice ingestion may be an environmental risk factor for hypertension or an apparent mineralocorticoid excess state in patients with a mutation in HSD11B2. In our patient, the exact dose of glycyrrhizic acid could not be known because she took the licorice in the herbal medicine shop without a prescription. Genetic screening might be necessary in some patients who display an excessive licorice response, as in our patient.

This case highlights the potential for cardiovascular complications to be associated with herbal medicines such as licorice. Clinicians should be aware that patients presenting to the emergency department with ventricular arrhythmia and uncertain hypokalemia should be questioned about licorice intake. Obtaining a detailed history is essential for patients admitted to the hospital for electrical storm.

\section{Acknowledgement}

This study was supported by research funds from Chosun University Hospital, 2017. 


\section{Conflict of interest}

- Authors: The authors declare that there is no conflict of interest.

- Reviewers: Nothing to declare

- Editors: Nothing to declare

\section{References}

1. Farese RV, Jr., Biglieri EG, Shackleton CH, Irony I, Gomez-Fontes R. Licorice-induced hypermineralocorticoidism. N Engl J Med 1991;325:12231227.

2. Walker BR, Edwards CR. Licorice-induced hypertension and syndromes of apparent mineralocorticoid excess. Endocrinol Metab Clin North Am 1994;23:359-377.

3. Kowey PR. An overview of antiarrhythmic drug management of electrical storm. Can J Cardiol 1996;12 Suppl B:3B-8B.

4. Cho S, Lim B, Cho H, Joung J, Choi Y, Kim D, et al. Licorice induced hypokalemia. Korean J Nephrol 2001;20:1021-1025.

5. Seo SC, Eom MS, Park WU, Go HI. Licorice-induced hypokalemia and myopathy. Korean J Nephrol 2006;25:651-656.

6. Kim BJ, Hong YH, Sung JJ, Park KS, Hyun CL, Choe GY, et al. A case of glycyrrhizin (licorice)-induced hypokalemic myopathy. J Korean Neurol Assoc 2006;24:389-391.

7. Hong HI, Han KH, Hwang JW, Kim YD, Shim MS, Kim JY. A case of licorice induced hypokalemic paralysis with rhabdomyolysis. J Korean Soc Endocrinol 2005;20:179-182.

8. Panduranga P, Al-Rawahi N. Licorice-induced severe hypokalemia with recurrent torsade de pointes. Ann Noninvasive Electrocardiol 2013;18:593596. doi: 10.1111/anec.12076.

9. Miyamoto K, Kawai H, Aoyama R, Watanabe H, Suzuki K, Suga N, et al. Torsades de pointes induced by a combination of garenoxacin and disopyramide and other cytochrome P450, family 3, subfamily a polypeptide4-influencing drugs during hypokalemia due to licorice. Clin Exp Nephrol 2010;14:164-167. doi: 10.1007/s10157-009-0244-9.

10. Crean AM, Abdel-Rahman SE, Greenwood JP. A sweet tooth as the root cause of cardiac arrest. Can J Cardiol 2009;25:e357-e358.

11. Eriksson JW, Carlberg B, Hillörn V. Life-threatening ventricular tachycardia due to liquorice-induced hypokalaemia. J Intern Med 1999;245:307310 .

12. Oztürk S, Karaman K, Cetin M, Erdem A. Polymorphic ventricular tachycardia (torsades de pointes) due to licorice root tea. Turk Kardiyol Dern Ars 2013;41:241-244. doi: 10.5543/tkda.2013.72547.

13. Park KA, Seok SJ, Kim SJ, Gil HW, Yang JO, Lee EY, et al. A case of licorice-Induced hypokalemic rhabdomyolysis in a patient using a diuretic drug. Korean J Med 2011;80:258-262.

14. Størmer FC, Reistad R, Alexander J. Glycyrrhizic acid in liquorice--evaluation of health hazard. Food Chem Toxicol 1993;31:303-312.

15. Russo S, Mastropasqua M, Mosetti MA, Persegani C, Paggi A. Low doses of liquorice can induce hypertension encephalopathy. Am J Nephrol 2000;20:145-148.

16. Harahap IS, Sasaki N, Gunadi, Yusoff S, Lee MJ, Morikawa S, et al. Herbal medicine containing licorice may be contraindicated for a patient with an HSD11B2 mutation. Evid Based Complement Alternat Med 2011;2011:646540. doi: 10.1093/ecam/nep211. 